\title{
Severe Acute Respiratory Infection Surveillance during the Initial Phase of the COVID-19 Outbreak in North India: A Comparison of COVID-19 to Other SARI Causes
}

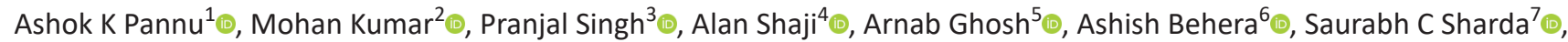

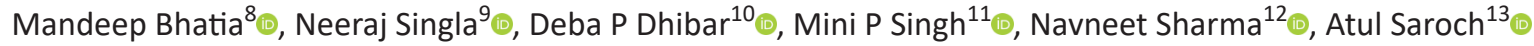

\begin{abstract}
Introduction: World Health Organization proposes severe acute respiratory infection (SARI) case definition for coronavirus disease 2019 (COVID-19) surveillance; however, early differentiation between SARI etiologies remains challenging. We aimed to investigate the spectrum and outcome of SARI and compare COVID-19 to non-COVID-19 causes.

Patients and methods: A prospective cohort study was conducted between March 15, 2020, to August 15, 2020, at an adult medical emergency in North India. SARI was diagnosed using a "modified" case definition-febrile respiratory symptoms or radiographic evidence of pneumonia or acute respiratory distress syndrome of $\leq 14$ days duration, along with a need for hospitalization and in the absence of an alternative etiology that fully explains the illness. COVID-19 was diagnosed with reverse transcription-polymerase chain reaction testing.

Results: In total, 95/212 (44.8\%) cases had COVID-19. Community-acquired pneumonia ( $n=57)$, exacerbation of chronic lung disease ( $n=11)$, heart failure $(n=11)$, tropical febrile illnesses $(n=10)$, and influenza A $(n=5)$ were common non-COVID-19 causes. No between-group differences were apparent in age $\geq 60$ years, comorbidities, oxygenation, leukocytosis, lymphopenia, acute physiology and chronic health evaluation (APACHE)-II score, CURB-65 score, and ventilator requirement at 24-hour. Bilateral lung distribution and middle-lower zones involvement in radiography predicted COVID-19. The median hospital stay was longer with COVID-19 ( 12 versus 5 days, $p=0.000$ ); however, mortality was similar (31.6\% versus $28.2 \%, p=0.593)$. Independent mortality predictors were higher mean APACHE II in COVID-19 and early ventilator requirement in non-COVID-19 cases.

Conclusions: COVID-19 has similar severity and mortality as non-COVID-19 SARI but requires an extended hospital stay. Including radiography in the SARI definition might improve COVID-19 surveillance.

Keywords: COVID-19, SARS-CoV-2, Severe acute respiratory infection, Severity, Surveillance.

Indian Journal of Critical Care Medicine (2021): 10.5005/jp-journals-10071-23882
\end{abstract}

\section{INTRODUCTION}

Severe acute respiratory infection (SARI), a World Health Organization (WHO) case surveillance definition, was initially introduced for complicated flu infection in 2014.' It is defined as febrile respiratory illness (fever and at least one symptom of respiratory disease, e.g., cough and shortness of breath) requiring hospitalization without an alternative diagnosis that fully explains the clinical presentation. ${ }^{1,2}$ Because of ease to use and excellent sensitivity, the definition has gained global acceptance to identify a catastrophic respiratory infection pandemic early in the course. ${ }^{1-3}$ Many contagious and rapidly progressive viral respiratory illnesses such as severe acute respiratory syndrome coronavirus (SARS-CoV), middle-east respiratory syndrome coronavirus, Ebola, Nipah have been contained at the source of origin with this robust surveillance strategy.

A new human coronavirus, called 2019 novel coronavirus or SARS-CoV-2 associated with severe respiratory disease, has emerged from Wuhan, China, in December 2019. ${ }^{4}$ Within a short period of a few weeks, the infection had resulted in a pandemic with high mortality. The symptoms of coronavirus disease 2019 (COVID-19) typically include fever, cough, myalgia or fatigue, and shortness of breath. ${ }^{5-8}$ Because this clinical spectrum is so overlapping with many respiratory or other infections, it remains challenging to achieve an early differential diagnosis of these diseases-the
1-4,6-10,12,13 Department of Internal Medicine, Postgraduate Institute of Medical Education and Research, Chandigarh, India

5,11 Department of Virology, Postgraduate Institute of Medical Education and Research, Chandigarh, India

Corresponding Author: Atul Saroch, Department of Internal Medicine, Postgraduate Institute of Medical Education and Research, Chandigarh, India, Phone: +91 7289873798, e-mail: atulsaroch@gmail.com

How to cite this article: Pannu AK, Kumar M, Singh P, Shaji A, Ghosh A, Behera A, et al. Severe Acute Respiratory Infection Surveillance during the Initial Phase of the COVID-19 Outbreak in North India: A Comparison of COVID-19 to Other SARI Causes. Indian J Crit Care Med 2021;25(7):761-767.

Source of support: Nil

Conflict of interest: None

primary step is to prevent the spread of the virus. Therefore, $\mathrm{WHO}$ has proposed to use the case definition of SARI to detect a suspect COVID-19. 9

The causes of SARI are influenced by various factors, including demographic and geographic characteristics, the host's immune status, and preventive strategies (e.g., immunization), which underscore the importance of local epidemiological data. Earlier diagnosis and prediction of severity could improve patient

(c) Jaypee Brothers Medical Publishers. 2021 Open Access This article is distributed under the terms of the Creative Commons Attribution 4.0 International License (https://creativecommons.org/licenses/by-nc/4.0/), which permits unrestricted use, distribution, and non-commercial reproduction in any medium, provided you give appropriate credit to the original author(s) and the source, provide a link to the Creative Commons license, and indicate if changes were made. The Creative Commons Public Domain Dedication waiver (http://creativecommons.org/publicdomain/zero/1.0/) applies to the data made available in this article, unless otherwise stated. 
outcomes by leading to proactive isolation and critical unit transfers, prompt treatment, and better allocation of limited resources. Therefore, this study aimed to investigate the spectrum, etiology, illness severity, and outcome of patients admitted with SARI during surveillance for suspected COVID-19 cases in an adult medical emergency of North India.

\section{Materials and Methods}

\section{Study Site}

An isolated ward for patients with SARI presentation was established at the adult medical emergency of the Department of Internal Medicine of the PGIMER, Chandigarh. This hospital is a tertiary care academic hospital that provides healthcare to a large population of North India and has more than 50000 adult patients visits to its medical emergency annually.

\section{Study Design}

This was a hospital-based prospective cohort study.

\section{Study Population and Period}

All consecutive patients aged 18 years and above with SARI were enrolled from March 15, 2020, to August 15, 2020, i.e., from the beginning of the spread of SARS-CoV-2 through the peak of the outbreak in North India.

\section{Case Definition}

The WHO definition of SARI includes acute onset fever and at least one symptom of respiratory disease (e.g., cough and shortness of breath), along with a need for hospitalization and in the absence of an alternative etiology that fully explains the illness. ${ }^{9}$ However, because both fever and respiratory symptoms may not be present in all suspected cases, we have modified the definition with any of fever or respiratory symptoms and radiographic evidence of pneumonia or acute respiratory distress syndrome (ARDS) with no identified cause. The WHO case definition for influenza surveillance
(2014) described acute illness as the symptom onset within the last 10 days; however, the duration was not explicitly mentioned for the COVID-19 surveillance definition of SARI (2020). ${ }^{1,9}$ Initial published reports describing clinical features of COVID-19 demonstrated that a median [interquartile range (IQR)] duration from symptom onset to shortness of breath was 8 days (5-13), and to ARDS was 9 days (8-14). ${ }^{5}$ Accordingly, a febrile respiratory illness of $\leq 14$ days duration was defined as SARI for COVID-19 surveillance in this study. We excluded patients with a history of international travel or contact with a confirmed COVID-19 case in the last 14 days before illness onset. Figure 1 shows our surveillance system, enrolment process, and flow of the patients.

A confirmed case of COVID-19 was defined as a positive result on real-time reverse transcriptase polymerase chain reaction (RT-PCR) assay for SARS-CoV-19 of the nasopharyngeal swab, throat swab, or endotracheal aspirate samples.

\section{Data Collection}

All consecutive patients fulfilling inclusion and exclusion criteria underwent a history taking, physical examination, and laboratory investigations, including RT-PCR for SARS-CoV-2, complete blood count (hemoglobin, leukocyte count, and platelets), biochemistry (serum sodium and potassium, blood urea and creatinine, bilirubin, liver transaminases), coagulation testing, arterial blood gas analysis with lactate, and chest radiograph. Polymerase chain reaction for H1N1 and other influenza viruses (A and B) were performed by following standard recommendations. ${ }^{10}$ Further investigations, including cultures of blood and body fluids, serological testing for tropical febrile illnesses, and thoracic computed tomography, were done according to the management of SARI. Chest radiographs (preferably a posteroanterior view) were obtained with the use of the digital radiographic system. Radiograph assessments were performed by one of the three authors (A.K.P., M.K., and A.S.) by documenting normal or abnormal, and if abnormal-lung distribution, the pattern of parenchymal lesions, and associated findings (e.g., pleural effusion).

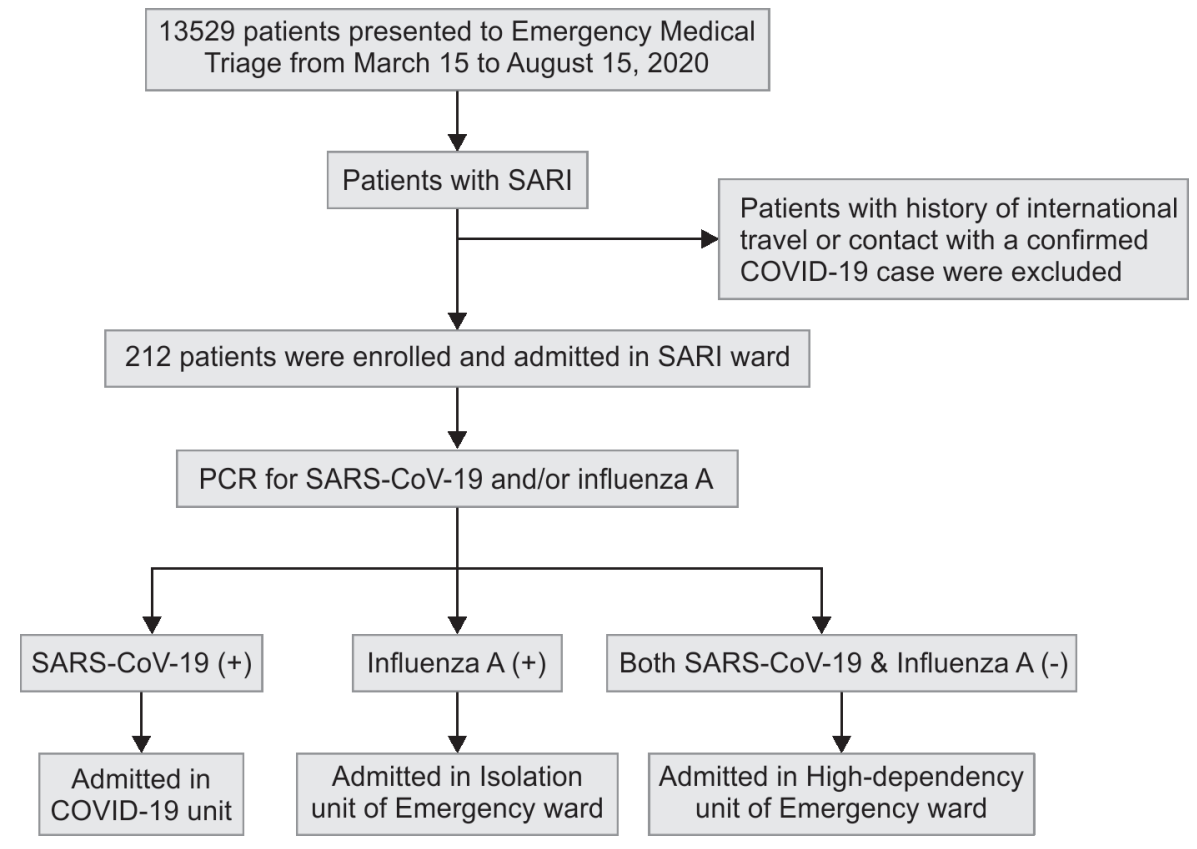

Fig. 1: Flowchart showing the SARI surveillance system, enrolment process, and flow of the patients 
We defined the degree of disease severity at the time of admission in four ways, according to critical illness severity score [acute physiology and chronic health evaluation II (APACHE II) score (range, 0-71; with higher scores indicating greater disease severity)], organ dysfunction [sequential organ failure assessment (SOFA) score (range, 0-20; with higher scores indicating more severe organ dysfunction) and quick SOFA (qSOFA) score (range, 0-3; with $\geq 2$ scores indicating severe organ dysfunction)], need of ICU admission in respiratory infections (CURB-65 score $\geq 2$ [range, 0-5; with scores $\geq 2$ indicating a high risk of mortality], and respiratory failure requiring invasive mechanical ventilation in first 24 hours [yes or no]), and shock requiring vasopressors in first 24 hours (yes or no). ${ }^{11-14}$ The diagnosis and treatment of SARI patients were following standard guidelines. ${ }^{10,13,15-21}$ COVID-19 management was modified according to the available evidence during the pandemic. Outcomes were measured as in-hospital mortality and length of hospital stay.

\section{Standard Protocol Approval}

The Institutional Ethics Committee approved the study (No.: INT/ IEC/2020/SPL-882). Written informed consent was waived in light of case surveillance among suspected COVID-19 patients during the pandemic with high mortality, the nature of the contagious illness, and the participant's isolation. We used anonymized patient data, and no intervention of any kind was performed. The waiver did not adversely affect the participant's rights and welfare, and, where appropriate, additional information was provided.

\section{Statistical Analysis}

All analyses were performed using Statistical Package for the Social Sciences (SPSS), version 25 for Mac. The patients were divided according to the etiology of SARI into COVID-19 and non-COVID-19. Categorical variables were summarized with frequencies and proportions and were compared with the use of the chi-square test. Continuous data were summarized with mean with standard deviation (SD) or median with IQR and compared with the use of Student's $t$-test or the nonparametric Mann-Whitney-Wilcoxon test. Multiple logistic regression analysis was used to identify independent predictors of death. All tests of significance were two-tailed, and a $p$-value of 0.05 or less was considered to indicate statistical significance.

\section{Results}

In total, 212 patients who fulfilled the "modified" case definition of SARI were enrolled from adjoining geographic regions of North India, including Punjab (35.5\%), Haryana (27.4\%), Chandigarh (24.5\%), Himachal Pradesh (6.1\%), Uttar Pradesh (5.7\%), Bihar (0.5\%), and New Delhi (0.5\%). Ninety-five (44.8\%) patients had confirmed SARS-CoV-2 infection. Among 117 non-COVID-19 cases, community-acquired pneumonia ( $n=57,26.9 \%$ ) was the most common diagnosis, next to being acute exacerbation of chronic lung disease ( $n=11,5.2 \%$ ), acute decompensated heart failure ( $n=11,5.2 \%)$, tropical febrile illnesses $(n=10,4.7 \%$; scrub typhus, $n=4)$, influenza A ( $n=5,2.4 \% ; \mathrm{H} 1 \mathrm{~N} 1, n=4)$, tuberculosis ( $n=4,1.9 \%)$, Pneumocystis jiroveci pneumonia $(n=4,1.9 \%)$, and Staphylococcus aureus bacteremia ( $n=2,0.9 \%)$, pyopneumothorax $(n=1)$, Escherichia coli urosepsis $(n=1)$, pulmonary thromboembolism $(n=1)$, amniotic fluid embolism $(n=1)$, malignant melanoma with lung metastasis $(n=1)$, acute promyelocytic leukemia $(n=1)$, acuteon-chronic liver failure $(n=1)$, and undiagnosed $(n=6,2.8 \%)$. A rapid surge of COVID-19 cases was noted from June 2020 (Fig. 2). Out of 95 COVID-19 cases, 78 fulfilled the WHO case definition of SARI (fever with respiratory symptoms). An additional 17 (17.9\%) patients were diagnosed with the inclusion of chest radiograph abnormalities (abnormal radiograph with respiratory symptoms, $n=16$; abnormal radiograph with fever, $n=1$ ). On the contrary, 105/117 non-COVID-19 cases fulfilled WHO case definition, 10 had abnormal radiograph with respiratory symptoms, and 2 had abnormal radiograph with fever.

The mean age was 50.3 years (range, 18-93 years), and COVID19 patients were older than non-COVID-19 patients by a mean of 6 years $(p=0.005$ ) (Table 1). Most clinical features were similar on admission, but chest radiograph abnormality $(p=0.036)$, including bilateral lung distribution ( $p=0.004)$, and involvement of both middle and lower zones $(p=0.003)$ were more frequent in COVID19. No between-group differences were apparent in leukocytosis, lymphopenia, and arterial blood gas parameters, including oxygenation (ratio of the arterial partial pressure of oxygen and the fraction of inspired oxygen); however, the non-COVID-19 group had a higher mean leukocyte count $(p=0.011)$, lower prothrombin index $(p=0.011)$, and higher serum bilirubin $(p=0.001)$. Illness severity

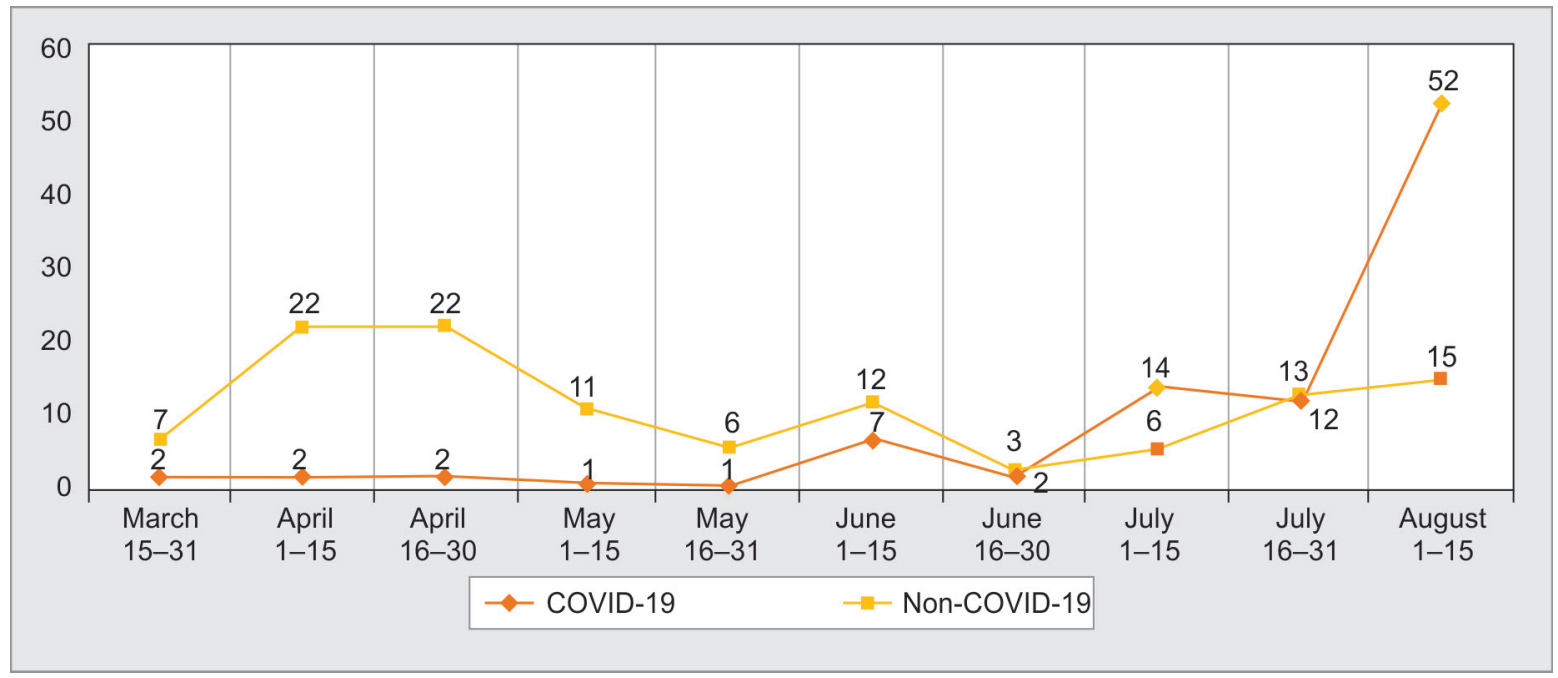

Fig. 2: Distribution of SARI cases through the five-month study period 
Table 1: Clinical, radiological, and laboratory features of SARI patients on presentation with a comparison between COVID-19 and non-COVID-19 groups

\begin{tabular}{|c|c|c|c|c|}
\hline Parameters & Total $(n=212)$ & COVID-19 $(n=95)$ & Non-COVID-19 $(n=117)$ & $p$-value \\
\hline Age (years), mean $\pm S D$ & $50.3 \pm 15.9$ & $53.7 \pm 13.4$ & $47.6 \pm 17.2$ & 0.005 \\
\hline Age $\geq 60$ years, $n(\%)$ & $70(33.0 \%)$ & $36(37.9 \%)$ & $34(29.1 \%)$ & 0.119 \\
\hline Male gender, $n(\%)$ & $126(59.4 \%)$ & $62(65.3 \%)$ & $64(54.7 \%)$ & 0.119 \\
\hline \multicolumn{5}{|l|}{ Medical comorbidities, $n(\%)$} \\
\hline None & 66 (31.1\%) & 26 (27.4\%) & 40 (34.2\%) & 0.286 \\
\hline Diabetes mellitus & $40(18.9 \%)$ & $20(21.1 \%)$ & $20(17.1 \%)$ & 0.464 \\
\hline Hypertension or coronary artery disease & $24(11.3 \%)$ & $15(15.8 \%)$ & $9(7.7 \%)$ & 0.064 \\
\hline Chronic kidney disease & $14(6.6 \%)$ & $6(6.3 \%)$ & $8(6.8 \%)$ & 0.879 \\
\hline Chronic lung disease & $13(6.1 \%)$ & $3(3.2 \%)$ & $10(8.5 \%)$ & 0.104 \\
\hline Others* & $38(17.9)$ & $18(18.9)$ & $20(17.1 \%)$ & 0.704 \\
\hline$\geq 2$ comorbidities & $17(8.0 \%)$ & $7(7.4 \%)$ & $10(8.5 \%)$ & 0.753 \\
\hline Duration of illness (days), median (IQR) & $5.0(3.0-7.0)$ & $5.0(3.0-7.0)$ & $5.0(3.0-7.0)$ & 0.657 \\
\hline \multicolumn{5}{|l|}{ Chief complaints, $n(\%)$} \\
\hline Shortness of breath & $201(94.8 \%)$ & $90(94.7 \%)$ & $111(94.9 \%)$ & 0.965 \\
\hline Fever & $179(84.4 \%)$ & $77(81.1 \%)$ & $102(87.2 \%)$ & 0.221 \\
\hline Cough & $125(59.0 \%)$ & $55(57.9 \%)$ & $70(59.8 \%)$ & 0.776 \\
\hline Sore throat & $23(10.8 \%)$ & $10(10.5 \%)$ & $13(11.1 \%)$ & 0.892 \\
\hline Altered mental status & $15(7.5 \%)$ & $6(6.3 \%)$ & $9(7.7 \%)$ & 0.697 \\
\hline Myalgia or headache & $19(9.0 \%)$ & $7(7.4 \%)$ & $12(10.3 \%)$ & 0.464 \\
\hline Diarrhea, abdominal pain or vomiting & $24(11.3 \%)$ & $9(9.5 \%)$ & $15(12.8 \%)$ & 0.444 \\
\hline Oxygen saturation <94\%, $n(\%)$ & $194(91.5 \%)$ & $83(87.4 \%)$ & $111(94.9 \%)$ & 0.051 \\
\hline Respiratory rate (per min), mean \pm SD & $26.5 \pm 5.1$ & $25.9 \pm 4.4$ & $27.0 \pm 5.6$ & 0.119 \\
\hline Pulse rate (per min), mean $\pm S D$ & $105.2 \pm 17.0$ & $102.4 \pm 14.6$ & $107.4 \pm 18.5$ & 0.031 \\
\hline Systolic blood pressure $(\mathrm{mm} \mathrm{Hg})$, mean $\pm \mathrm{SD}$ & $118.8 \pm 21.6$ & $121.2 \pm 20.4$ & $116.8 \pm 22.5$ & 0.146 \\
\hline Diastolic blood pressure $(\mathrm{mm} \mathrm{Hg})$, mean $\pm S D$ & $73.9 \pm 14.1$ & $75.2 \pm 10.7$ & $72.8 \pm 16.4$ & 0.229 \\
\hline Score on Glasgow coma scale $<15, n(\%)$ & $26(12.3 \%)$ & $8(8.4 \%)$ & $18(15.4 \%)$ & 0.124 \\
\hline Chest radiography, $n$ (\%) & $(n=197)$ & $(n=90)$ & $(n=107)$ & \\
\hline Abnormal & $179(90.9 \%)$ & $86(95.6 \%)$ & $93(86.9 \%)$ & 0.036 \\
\hline Bilateral lung distribution & $152(77.2 \%)$ & $80(93.0 \%)$ & $72(77.4 \%)$ & 0.004 \\
\hline \multicolumn{5}{|l|}{ Predominant zone involvement } \\
\hline Upper & $4(2.1 \%)$ & $1(1.1 \%)$ & $3(2.8 \%)$ & 0.420 \\
\hline Middle & $20(10.3 \%)$ & $8(9.2 \%)$ & $12(11.2 \%)$ & 0.645 \\
\hline Lower & $38(19.6 \%)$ & $16(18.4 \%)$ & $22(20.6 \%)$ & 0.705 \\
\hline Middle and Lower & $61(31.4 \%)$ & $37(42.5 \%)$ & $24(22.4 \%)$ & 0.003 \\
\hline Diffuse & $59(30.4)$ & $22(25.3 \%)$ & $37(34.6 \%)$ & 0.162 \\
\hline \multicolumn{5}{|l|}{ Predominant parenchymal lesions } \\
\hline Multifocal alveolar opacities & $74(42.8 \%)$ & $30(38.0 \%)$ & $44(46.8 \%)$ & 0.242 \\
\hline Reticulonodular infiltrates & $72(41.6 \%)$ & $35(44.3 \%)$ & $37(39.4 \%)$ & 0.511 \\
\hline Lobar consolidation & $20(11.6 \%)$ & $10(12.7 \%)$ & $10(10.6 \%)$ & 0.679 \\
\hline Cavitation & $6(3.5 \%)$ & $4(5.1 \%)$ & $2(2.1 \%)$ & 0.293 \\
\hline Pleural effusion & $20(10.2 \%)$ & $5(5.6 \%)$ & $15(14.0 \%)$ & 0.079 \\
\hline Total leukocyte counts (per $\mu \mathrm{L}$ ), median (IQR) & $9700(7200-13,500)$ & $8800(6400-12,400)$ & $10,450(7925-14,225)$ & 0.011 \\
\hline Leukocytosis (>11,000 per $\mu \mathrm{L}), n(\%)$ & $77(36.3 \%)$ & $29(30.5 \%)$ & $48(41.0 \%)$ & 0.103 \\
\hline Neutrophil-lymphocyte ratio, median (IQR) & $7.3(3.9-12.6)$ & $7.6(3.7-12.9)$ & $7.3(4.1-12.4)$ & 0.713 \\
\hline Lymphopenia (<1500 per $\mu \mathrm{L}), \mathrm{n}(\%)$ & $137(68.8 \%)$ & $68(71.6 \%)$ & $69(59.0 \%)$ & 0.100 \\
\hline Platelets (per $\mu \mathrm{L}$ ), median (IQR) & $189,000(107,000-259,000)$ & $194,000(147,000-271,000)$ & $178,500(88,250-258,250)$ & 0.121 \\
\hline Prothrombin index (\%), median (IQR) & $88.0(77.0-97.0)$ & $91.0(84.2-99.0)$ & $82.0(73.0-93.5)$ & 0.000 \\
\hline Serum sodium $(\mathrm{mEq} / \mathrm{L})$, mean $\pm \mathrm{SD}$ & $136.1 \pm 7.0$ & $136.2 \pm 5.3$ & $136.0 \pm 8.1$ & 0.869 \\
\hline Blood urea $(\mathrm{mg} / \mathrm{dL})$, median (IQR) & $47.5(29.0-79.7)$ & $43.5(29.0-72.7)$ & $51.0(30.0-91.7)$ & 0.197 \\
\hline Serum creatinine (mg/dL), median (IQR) & $1.0(0.7-1.8)$ & $0.9(0.7-1.4)$ & $1.2(0.7-2.6)$ & 0.099 \\
\hline Serum bilirubin (mg/dL), median (IQR) & $0.6(0.4-1.0)$ & $0.5(0.3-0.7)$ & $0.7(0.4-1.3)$ & 0.001 \\
\hline Aspartate transaminase (U/L), median (IQR) & $46.0(28.0-75.0)$ & $52.5(32.7-66.7)$ & $41.0(26.0-86.0)$ & 0.171 \\
\hline Alanine transaminase (U/L), median (IQR) & $40.0(19.5-68.5)$ & $42.5(21.7-69.7)$ & $36.0(19.0-63.0)$ & 0.262 \\
\hline Blood $\mathrm{pH}$, mean \pm SD & $7.37 \pm 0.11$ & $7.37 \pm 0.12$ & $7.38 \pm 0.11$ & 0.705 \\
\hline $\mathrm{PO}_{2}: \mathrm{FiO}_{2}$ ratio $(\mathrm{mmHg})$, mean $\pm \mathrm{SD}$ & $227.9 \pm 105.9$ & $232.4 \pm 110.8$ & $224.9 \pm 102.9$ & 0.661 \\
\hline Lactate $(\mathrm{mmol} / \mathrm{L})$, median $(\mathrm{IQR})$ & $2.0(1.5-2.8)$ & $2.0(1.3-2.7)$ & $2.1(1.6-2.9)$ & 0.167 \\
\hline
\end{tabular}

*Others include chronic hematological disorder (8), morbid obesity (body mass index $\left.\geq 40 \mathrm{~kg} / \mathrm{m}^{2}\right)(n=6)$, malignancy $(n=6)$, postrenal transplantation $(n=5)$, chronic immunosuppressive use $(n=5)$, pregnancy $(n=4)$, liver cirrhosis $(n=3)$, and human immunodeficiency virus $(n=1)$; $\mathrm{FiO}_{2}$, fraction of inspired oxygen; $\mathrm{PaO}_{2}$, arterial partial pressure of oxygen 
at admission in terms of APACHE II score, CURB-65 score, and ventilator requirement at 24 hours was similar, but non-COVID-19 patients were more likely than COVID-19 patients to have higher values of SOFA score $(p=0.000)$ and qSOFA score $(p=0.022)$, and need of vasopressor support for shock ( $p=0.029$ ) (Table 2$)$. The median length of hospital stay was 7 days (range, 1-72 days). The duration was significantly longer in COVID-19 patients (12 days versus 5 days, $p=0.000$ ).

The case fatality rate was $28.9 \%$, with no statistical difference between COVID-19 and non-COVID-19 patients (31.6\% versus $28.2 \%, p=0.593$ ) (Table 2). Univariate analysis showed that the risk of death in COVID-19 patients was increased among those who had a higher pulse rate $(p=0.047)$, higher respiratory rate $(p=0.000)$, leukocytosis ( $p=0.014)$, increased neutrophil-lymphocyte ratio ( $p=0.047)$, elevated blood urea $(p=0.000)$, high APACHE II score $(p=0.000)$, high SOFA score $(p=0.004)$, qSOFA score $\geq 2(p=0.050)$, CURB-65 score $\geq 2(p=0.000)$, and the need of invasive ventilation within 24 hours $(p=0.000)$. However, in multivariate analysis, high APACHE II score was the only predictor of death $(p=0.028)$ (Table 3). For non-COVID-19 patients, the factors predicted mortality on univariate analysis were a higher pulse rate $(p=0.028)$, higher respiratory rate $(p=0.000)$, increased neutrophil-lymphocyte ratio $(p=0.005)$, high APACHE II score $(p=0.017)$, high SOFA score $(p=0.010)$, qSOFA score $\geq 2(p=0.027)$, CURB-65 score $\geq 2(p=0.000)$, the need of invasive ventilation $(p=0.000)$, and the need of vasopressors ( $p=0.002$ ). Among these, only the requirement of invasive ventilation was found to be the independent predictor of death on the multivariant regression analysis $(p=0.038)$ (Table 3 ).

\section{Discussion}

Our study represents a model of enhanced surveillance strategy at a medical emergency during the initial phase of a respiratory infection outbreak. This report of 212 cases from North India is unique in its description of comparison between COVID-19 patients and other non-COVID-19 diseases (infectious and noninfectious), both diagnosed as SARI at presentation using a "modified" case definition and had similar clinical characteristics and illness severity. Abnormal chest radiograph with bilateral distribution or both middle and lower zones involvement favored COVID-19 diagnosis. Mortality was high in both groups, but COVID19 required prolonged hospitalization.

A high prevalence of medical comorbidities, particularly diabetes and cardiovascular conditions, was documented in SARI patients. Difficulty breathing was the most common presenting symptom, and the absence of fever was not infrequent, more so with COVID-19. Expanding WHO's case definition of SARI to include radiographic abnormalities was appropriate in our study. It detected approximately $18 \%$ additional COVID-19 patients as well as predicted a COVID-19 diagnosis that could enable early isolation. COVID-19 patients less commonly reported abdominal symptoms, headache, myalgia, and altered sensorium; however, because this cohort represents the more severe end of COVID-19, we cannot describe the full spectrum of the disease. ${ }^{5-8,22-24}$

Non-COVID-19 causes like community-acquired pneumonia, acute decompensation or exacerbation of underlying chronic cardiorespiratory conditions, endemic infections such as scrub

Table 2: Illness severity at presentation and outcomes of SARI patients with a comparison between COVID-19 and non-COVID-19 groups

\begin{tabular}{|c|c|c|c|c|}
\hline Parameters & Total $(n=212)$ & $\operatorname{COVID}-19(n=95)$ & Non-COVID-19 $(n=117)$ & $p$-value \\
\hline APACHE II score, mean \pm SD & $14.0 \pm 6.8$ & $13.5 \pm 7.3$ & $14.3 \pm 6.4$ & 0.483 \\
\hline SOFA score, mean \pm SD & $4.4 \pm 2.6$ & $3.5 \pm 2.3$ & $4.9 \pm 2.7$ & 0.000 \\
\hline qSOFA score $\geq 2, n(\%)$ & $59(27.8 \%)$ & $19(20.0 \%)$ & $40(34.2 \%)$ & 0.022 \\
\hline CURB-65 score $\geq 2, n$ (\%) & $75(35.5 \%)$ & $28(29.8 \%)$ & $47(40.9 \%)$ & 0.097 \\
\hline Need of invasive mechanical ventilation at $24 \mathrm{hr}, n(\%)$ & $39(18.4 \%)$ & $14(14.7 \%)$ & $25(21.4 \%)$ & 0.215 \\
\hline Need of vasopressor support at $24 \mathrm{hr}, n(\%)$ & $19(9.0 \%)$ & $4(4.2 \%)$ & $15(12.8 \%)$ & 0.029 \\
\hline In-hospital mortality, $n$ (\%) & $63(28.9 \%)$ & $30(31.6 \%)$ & $33(28.2 \%)$ & 0.593 \\
\hline Hospital stay (days), median (IQR) & $7.0(3.0-13.0)$ & $12.0(7.0-17.0)$ & $5.0(1.0-8.5)$ & 0.000 \\
\hline
\end{tabular}

Table 3: Multivariate logistic regression analysis for predictors of mortality in COVID-19 and non-COVID-19 groups

\begin{tabular}{|c|c|c|c|c|c|c|}
\hline \multirow[b]{2}{*}{ Parameters } & \multicolumn{3}{|l|}{ COVID-19 $(n=95)$} & \multicolumn{3}{|c|}{ Non-COVID-19 $(n=117)$} \\
\hline & Died $(n=30)$ & Survived $(n=65)$ & $p$-value & Died $(n=33)$ & Survived $(n=84)$ & $p$-value \\
\hline Respiratory rate (per min), mean \pm SD & $28.1 \pm 4.4$ & $24.8 \pm 3.9$ & 0.803 & $30.3 \pm 6.5$ & $25.7 \pm 4.5$ & 0.470 \\
\hline Pulse rate (per min), mean $\pm S D$ & $106.6 \pm 13.8$ & $100.3 \pm 14.7$ & 0.337 & $113.6 \pm 19.8$ & $105.1 \pm 17.6$ & 0.172 \\
\hline Leukocytosis $(\geq 11,000 / \mu \mathrm{L}), n(\%)$ & $15(47.9 \%)$ & $14(22.2 \%)$ & 0.593 & $13(41.9 \%)$ & $35(41.2 \%)$ & $-{ }^{\mathrm{a}}$ \\
\hline Neutrophil-lymphocyte ratio, median (IQR) & $10.7(5.3-15.0)$ & $6.2(3.6-10.2)$ & 0.708 & $10.0(6.8-15.0)$ & $6.0(3.6-10.1)$ & 0.066 \\
\hline Blood urea (mg/dL), median (IQR) & $65.5(45.0-116.5)$ & $40.5(28.0-66.0)$ & 0.487 & $60.0(43.0-108.0)$ & $48.0(28.0-85.0)$ & $--^{\mathrm{a}}$ \\
\hline APACHE II score, mean \pm SD & $18.4 \pm 6.2$ & $11.2 \pm 6.7$ & 0.028 & $16.9 \pm 5.1$ & $13.4 \pm 6.6$ & 0.636 \\
\hline SOFA score, mean \pm SD & $4.7 \pm 3.0$ & $2.9 \pm 1.7$ & 0.960 & $6.1 \pm 2.0$ & $4.5 \pm 2.7$ & 0.867 \\
\hline qSOFA score $\geq 2, n(\%)$ & $10(31.2 \%)$ & $9(14.3 \%)$ & 0.414 & $16(50.0 \%)$ & $24(28.2 \%)$ & 0.253 \\
\hline CURB-65 score $\geq 2, n(\%)$ & $18(58.1 \%)$ & $10(15.9 \%)$ & 0.093 & $21(67.7 \%)$ & $26(30.9 \%)$ & 0.645 \\
\hline Need of invasive ventilation, $n(\%)$ & $12(37.5 \%)$ & $2(3.2 \%)$ & 0.200 & 19 (59.4\%) & $6(7.1 \%)$ & 0.038 \\
\hline Need of vasopressor support, $n(\%)$ & $4(12.5 \%)$ & 0 & - & $9(2.8 \%)$ & $6(7.1 \%)$ & 0.743 \\
\hline
\end{tabular}

${ }^{a}$ Not included in multivariate analysis as univariant analysis did not show a statistical difference 
typhus or tuberculosis, sepsis from nonrespiratory infections, posed considerable diagnostic confusion. To differentiate COVID-19 from its counterpart early, coexisting conditions, clinical features, oxygenation, and early ventilator requirement did not help. However, COVID-19 patients had more prominent radiographic abnormalities. Finding the bilaterally distributed lesions with middle and lower zones' involvement might serve as an alert to initiate COVID-19 management, i.e., isolation, dexamethasone, anticoagulation, or remdesivir in a patient admitted with SARI in the medical emergency. ${ }^{25,26}$ Computed tomography has higher sensitivity and less interobserver variability than radiography; however, cost, radiation, and no wide-availability limit its screening role. Non-COVID-19 patients had a higher mean of SOFA, qSOFA $\geq 2$ in more than one-third, and shock requiring pressor support; therefore, they were more likely to have sepsis or sepsis-like syndrome with multiorgan dysfunction on admission. ${ }^{13}$

Both groups had about 30\% in-hospital mortality. After multivariable adjustment, mortality was associated with a higher APACHE II score in the COVID-19 group and a ventilator requirement at 24 hours in the non-COVID-19 group. Despite similar illness severity and case fatality, COVID-19 patients required a median hospital stay of more than double than non-COVID-19 patients. The rising global threat of reducing inpatient bed capacity due to the extended hospital stay of COVID-19 patients would be a significant concern in low- and middle-income countries, which emphasizes the need for healthcare reform to provide balanced and appropriate critical care services (e.g., ICU bed or ventilator) to both COVID-19 and non-COVID-19 patients. ${ }^{27-29}$

\section{Limitations}

Our study's major limitations are single-center small population size and a lack of detailed data regarding mechanical ventilation beyond the initial 24 hours and other major complications during hospitalization. We did not evaluate the possible causes of the prolonged hospitalization in COVID-19 patients, such as institutional isolation or discharge policies or a requirement of prolonged mechanical ventilation or oxygen supplementation because of nonresolving lung parenchymal disease, pulmonary thromboembolism, and/or lack of evidence-based treatment at the beginning of the pandemic. Certain potential markers associated with COVID-19 severity, such as D-dimer, C-reactive protein, serum ferritin, interleukin-6 levels, cardiac biomarkers, viral load, were not measured in all patients. ${ }^{30,31}$

\section{CONCLUSION}

The study contributes information to understanding the early triaging of patients with severe acute lower respiratory illness during the pandemic. Illness severity and mortality were high and comparable between COVID-19 and non-COVID-19 cases with SARI presentation; however, hospital stay was far more extended with a greater occupancy of the inpatient beds in the former. Including chest radiography into the WHO SARI definition might improve a COVID-19 surveillance model in a hospital setting.

\section{ACKnowledgment}

The authors thank Mrs. Sunaina Verma for her help with statistics.

\section{Author Contributions}

A.K.P.-conceived the idea, patient management, collected patient data, wrote the first draft, and revised the manuscript. M.K., P.S., A.S., A.G., and A.B.- patient management and collected patient data. S.C.S., M.B., N.S., D.P.D., M.P.S., and N.S.- patient management. A.S.- patient management, collected patient data, and revised the manuscript.

\section{ORCID}

Ashok K Pannu (1) https://orcid.org/0000-0002-4476-3478 Mohan Kumar (10 https://orcid.org/0000-0003-4426-9610 Pranjal Singh (1) https://orcid.org/0000-0001-9792-9163 Alan Shaji $\odot$ https://orcid.org/0000-0001-8027-6952 Arnab Ghosh (1) https://orcid.org/0000-0002-9628-6052 Ashish Behera (1) https://orcid.org/0000-0002-1750-2352 Saurabh C Sharda @ https://orcid.org/0000-0001-7039-0276 Mandeep Bhatia — https://orcid.org/0000-0002-6286-1231 Neeraj Singla 나 https://orcid.org/0000-0002-7983-1637 Deba P Dhibar (1) https://orcid.org/0000-0002-0201-0160 Mini P Singh (1) https://orcid.org/0000-0001-6263-1850 Navneet Sharma $\odot$ https://orcid.org/0000-0001-5707-9686 Atul Saroch ๑ https://orcid.org/0000-0001-9723-6500

\section{References}

1. World Health Organization. WHO surveillance case definitions for ILI and SARI: case definitions for influenza surveillance. Geneva: World Health Organization; 2014. Available at: https://www.who. int/influenza/surveillance_monitoring/ili_sari_surveillance_case_ definition/en/ (accessed March 30, 2021).

2. Fitzner J, Qasmieh S, Mounts AW, Alexander B, Besselaar T, Briand $S$, et al. Revision of clinical case definitions: influenza-like illness and severe acute respiratory infection. Bull World Health Organ 2018;96(2):122-128. DOI: 10.2471/BLT.17.194514.

3. World Health Organization. Pandemic influenza risk management: a WHO guide to inform \& harmonize national \& international pandemic preparedness and response. Geneva: World Health Organization (WHO); 2017. Available at: https://apps.who.int/iris/ handle/10665/259893. Accessed November 16, 2019.

4. Zhu N, Zhang D, Wang W, Li X, Yang B, Song J, et al. A novel coronavirus from patients with pneumonia in China, 2019. N Engl J Med 2020;382(8):727-733. DOI: 10.1056/NEJMoa2001017.

5. Huang C, Wang Y, Li X, Ren L, Zhao J, Hu Y, et al. Clinical features of patients infected with 2019 novel coronavirus in Wuhan, China. Lancet 2020;395(10223):497-506. DOI: 10.1016/S0140-6736(20)30183-5.

6. Guan WJ, Ni ZY, Hu Y, Liang WH, Ou CQ, He JX, et al. Clinical characteristics of coronavirus disease 2019 in China. N Engl J Med 2020;382(18):1708-1720. DOI: 10.1056/NEJMoa2002032.

7. Gandhi RT, Lynch JB, Del Rio C. Mild or moderate Covid-19. N Engl J Med 2020;383:1757-1766. DOI: 10.1056/NEJMcp2009249.

8. Berlin DA, Gulick RM, Martinez FJ. Severe Covid-19. N Engl J Med 2020;383:2451-2460. DOI: 10.1056/NEJMcp2009575.

9. World Health Organization. Global surveillance for COVID-19 caused by human infection with COVID-19 virus: interim guidance. Geneva: World Health Organization; 2020. Available at: https://apps. who.int/iris/bitstream/handle/10665/331506/WHO-2019-nCoVSurveillanceGuidance-2020.6-eng.pdf?sequence=1\&isAllowed=y. Accessed March 30, 2021.

10. Uyeki TM, Bernstein HH, Bradley JS, Englund JA, File TM, Fryet AM, et al. Clinical practice guidelines by the infectious diseases Society 
of America: 2018 update on diagnosis, treatment, chemoprophylaxis, and institutional outbreak management of seasonal influenzaa. Clin Infect Dis 2019;68(6):895-902. DOI: 10.1093/cid/ciy866.

11. Knaus WA, Draper EA, Wagner DP, Zimmerman JE. APACHE II: a severity of disease classification system. Crit Care Med 1985;13(10):818-829. Available at: https://pubmed.ncbi.nlm.nih.gov/3928249/

12. Vincent JL, Moreno R, Takala J, Mendonça AD, Bruining H, Reinhart CK, et al. The SOFA (sepsis-related organ failure assessment) score to describe organ dysfunction/failure. Intensive Care Med 1996;22:707710. DOI: $10.1007 / \mathrm{BF} 01709751$.

13. Singer M, Deutschman CS, Seymour CW, Shankar-Hari M, Annane $D$, Bauer $\mathrm{M}$, et al. The third international consensus definitions for sepsis and septic shock (sepsis-3). JAMA 2016;315:801-810. DOI: 10.1001/ jama.2016.0287.

14. Lim WS, Baudouin SV, George RC, Hill AT, Jamieson C, Jeune IL, et al. BTS guidelines for the management of community acquired pneumonia in adults: update 2009. Thorax 2009;64(suppl 3): iii1-iii55. DOI: $10.1136 /$ thx.2009.121434.

15. Bhimraj A, Morgan RL, Shumaker AH, Lavergne V, Baden L, Cheng VC, et al. Infectious diseases society of america guidelines on the treatment and management of patients with COVID-19. Clin Infect Dis 2020;2020:ciaa478. DOI: 10.1093/cid/ciaa478.

16. World Health Organization. Clinical management of COVID-19: interim guidance. Geneva: World Health Organization; 2020. Available at: https://www.who.int/publications/i/item/clinical-management-ofsevere-acute respiratory-infection-when-novel-coronavirus-(ncov)infection-is-suspected. Accessed May 30, 2020.

17. Ministry of health \& family Welfare, Government of India. Guidelines on Clinical Management of COVID - 19. New Delhi: Directorate General of Health Services. Ministry of Health and Family Welfare; 2020. Available at: https://www.mohfw.gov.in/ pdf/ClinicalManagementProtocolforCOVID19dated27062020.pdf. Accessed June 28, 2020.

18. Metlay JP, Waterer GW, Long AC, Anzueto A, Brozek J, Crothers K, et al. Diagnosis and treatment of adults with community-acquired pneumonia. Am J Respir Crit Care Med 2019;200(7):e45-e67. DOI: 10.1164/rccm.201908-1581ST.

19. Vogelmeier CF, Criner GJ, Martinez FJ, Anzueto A, Barnes PJ, Bourbeau $J$, et al. Global strategy for the diagnosis, management, and prevention of chronic obstructive lung disease 2017 report. GOLD executive summary. Am J Respir Crit Care Med 2017;195(5):557-582. DOI: 10.1164/rccm.201701-0218PP.

20. Yancy CW, Jessup M, Bozkurt B, Butler J, Casey DE Jr, Colvin MM, et al. 2017 ACC/AHA/HFSA focused update of the 2013 ACCF/ AHA guideline for the management of heart failure: a report of the American College of Cardiology/American Heart Association Task Force on Clinical Practice Guidelines and the Heart Failure Society of America. Circulation 2017;136(6):e137-e161. DOI: 10.1161/ CIR.0000000000000509.
21. Central TB Division, Ministry of Health \& Family Welfare, Government of India. Guidelines: technical and operational guidelines for TB control in India 2016. New Delhi:Central TB Division, Ministry of Health and Family Welfare, Government of India; 2018. Available at: https://tbcindia.gov. in $/$ index1.php? sublinkid $=4573 \&$ level $=2 \&$ lid $=3177 \&$ lang $=1$. Accessed May 3, 2019.

22. Docherty AB, Harrison EM, Green CA, Hardwick HE, Pius R, Norman $L$, et al. Features of 20133 UK patients in hospital with covid-19 using the ISARIC WHO Clinical Characterisation Protocol: prospective observational cohort study BMJ 2020;369:m1985. DOI: 10.1136/bmj. m1985.

23. Menni, C, Valdes AM, Freidin MB, Sudre $C H$, Nguyen $L H$, Drew DA, et al. Real-time tracking of self-reported symptoms to predict potential COVID-19. Nat Med 2020;26:1037-1040. DOI: 10.1038/s41591-0200916-2.

24. Parasa S, Desai M, Thoguluva CV, Patel HK, Kennedy KF, Roesch T, et al. Prevalence of gastrointestinal symptoms and fecal viral shedding in patients with coronavirus disease 2019: a systematic review and meta-analysis. JAMA Netw Open 2020;3(6):e2011335. DOI: 10.1001/ jamanetworkopen.2020.11335.

25. Rubin GD, Ryerson CJ, Haramati LB, Sverzellati N, Kanne JP, Raoof $S$, et al. The role of chest imaging in patient management during the COVID-19 pandemic: a multinational consensus statement from the Fleischner society. Chest 2020;158(1):106-116. DOI: 10.1016/j. chest.2020.04.003.

26. Korevaar DA, Kootte RS, Smits LP, van den Aardweg JG, Bonta PI, Schinkel J, et al. Added value of chest computed tomography in suspected COVID-19: an analysis of 239 patients. Eur Respir J 2020;56(2):2001377. DOI: 10.1183/13993003.01377-2020.

27. Rees EM, Nightingale ES, Jafari Y, Waterlow NR, Clifford S, Pearson CAB, et al. COVID-19 length of hospital stay: a systematic review and data synthesis. BMC Med 2020;18(1):270. DOI: 10.1186/s12916-020-01726-3.

28. Sprung CL, Joynt GM, Christian MD, Truog RD, Rello J, Nates $\mathrm{JL}$, et al. Adult ICU triage during the coronavirus disease 2019 pandemic: who will live and who will die? Recommendations to improve survival. Crit Care Med 2020;48(8):1196-1202. DOI: 10.1097/ CCM.0000000000004410.

29. Lewnard JA, Liu VX, Jackson ML, Schmidt MA, Jewell BL, Flores JP, et al. Incidence, clinical outcomes, and transmission dynamics of severe coronavirus disease 2019 in California and Washington: prospective cohort study. BMJ 2020;369:m1923. DOI: 10.1136/bmj.m1923.

30. Wu C, Chen X, Cai Y, Xia J, Zhou X, Xu S, et al. Risk factors associated with acute respiratory distress syndrome and death in patients with coronavirus disease 2019 pneumonia in Wuhan, China. JAMA Intern Med 2020;180(7):934-943. DOI: 10.1001/jamainternmed.2020.0994.

31. Shi S, Qin M, Shen B, Cai Y, Liu T, Yang F, et al. Association of cardiac injury with mortality in hospitalized patients with COVID-19 in Wuhan, China. JAMA Cardiol 2020;5(7):802-810. DOI: 10.1001/ jamacardio.2020.0950. 